\title{
Belphégor
}

\section{Fantômas - À l'ombre de la guillotine (Louis Feuillade, 1913) ou Quand le cinéma s'émancipe...}

\section{Bérengère Vachonfrance-Levet}

\section{(2) OpenEdition}

\section{Journals}

Édition électronique

URL : http://journals.openedition.org/belphegor/115

DOI : 10.4000/belphegor.115

ISSN : 1499-7185

Éditeur

LPCM

Référence électronique

Bérengère Vachonfrance-Levet, " Fantômas - À l'ombre de la guillotine (Louis Feuillade, 1913) ou Quand le cinéma s'émancipe... », Belphégor [En ligne], 11-1 | 2013, mis en ligne le 07 juillet 2013, consulté le 20 juin 2020. URL : http://journals.openedition.org/belphegor/115 ; DOl : https://doi.org/10.4000/ belphegor. 115

Ce document a été généré automatiquement le 20 juin 2020.

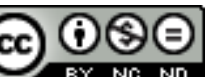

Belphégor est mis à disposition selon les termes de la Licence Creative Commons Attribution - Pas d'Utilisation Commerciale - Pas de Modification 4.0 International. 


\title{
Fantômas - À l'ombre de la guillotine (Louis Feuillade, 1913) ou Quand le cinéma s'émancipe...
}

\author{
Bérengère Vachonfrance-Levet
}

1 L'adaptation cinématographique par Louis Feuillade (1913-1914) de cinq volumes de la série romanesque des Fantômas de Pierre Souvestre et Marcel Allain, témoigne d'un moment charnière de l'histoire de la culture de masse en France, et pas seulement parce qu'interrompue par la Première Guerre Mondiale, elle marque la fin d'une (Belle-)époque, celle de l'hégémonie mondiale du cinéma français.

2 Bien plutôt, elle illustre un double mouvement d'émancipation du jeune cinéma à l'égard, d'une part, de la littérature populaire garante de succès et source d'inspiration privilégiée, et d'autre part, du théâtre, autre grand - et traditionnel, lui - spectacle de masse.

3 À cet égard, Fantômas - À l'ombre de la guillotine (1913), premier film adapté, est tout à fait significatif de ce double mouvement d'émancipation. Si Louis Feuillade prend soin de l'adosser au roman de Pierre Souvestre et Marcel Allain, il n'hésite pas à s'affranchir de l'œuvre littéraire originale lui opposant par exemple une narration linéaire proprement cinématographique, quitte à se tourner vers le théâtre et son esthétique pour répondre visuellement à des procédés littéraires. Mais, et alors même que l'on débat sur la nature du spectacle cinématographique autour de la question de la censure, Feuillade répond à sa façon, affirmant de fait l'autonomie du cinéma par rapport au théâtre, autre divertissement de masse, autre grand spectacle populaire de l'époque. 


\section{Fantômas sur les traces de Zigomar (La littérature populaire, un enjeu commercial pour les studios)}

4 La première adaptation cinématographique de Fantômas, celle réalisée par Louis Feuillade, est d'abord le fruit de la concurrence commerciale que les principales firmes françaises de production de films - elles-mêmes sous la menace grandissante des sociétés américaines - se livrent, tout particulièrement autour de l'adaptation des plus grands succès de la littérature populaire. Cette forme littéraire s'est certes transformée tout au long du XIXe siècle, mais elle reste tout aussi populaire. Il est donc naturel que le cinéma naissant (1895), lui aussi soumis, dès le départ, aux contraintes économiques et commerciales (ne s'agit-il pas d'une industrie ${ }^{1}$ ?) se tourne vers cette grande sœur pour $\mathrm{y}$ puiser les recettes du succès. On peut distinguer deux sources principales d'inspiration autour d'une même veine policière qui tend à se constituer en genre.

La première est d'inspiration américaine, et tout commence en 1908 lorsque VictorinHippolyte Jasset réalise pour la société Éclair l'adaptation d'un dime novel ${ }^{2}$ américain des années $1890^{3}$ : Nick Carter, le roi des détectives (6 épisodes), devenant ainsi, pour la postérité, le père du film policier français. Pathé réplique par la parodie, en 1911, avec une série de films réalisée par Paul Garbagni, et dont le héros est également un détective : Nick Winter. Puis très vite, Pathé fait appel à un feuilletoniste de renom, Gustave le Rouge, pour trois films: Charley Colms, Le Club des Élégants, Le Collier de la Danseuse (1912). De son côté, la société des films Éclipse produit, en 1911, une série de films autour d'un personnage de détective privé d'une série de romans américains, Nat Pinkerton, réalisée par Pierre Bressol, l'acteur qui incarnait Nick Carter à l'écran. Et la société Gaumont, elle, propose la série Main de Fer réalisée par Léonce Perret (trois épisodes entre 1912 et 1913) tandis que Louis Feuillade met en scène un détective, Dervieux (qu'incarne René Navarre) dans quatre films ${ }^{4}$. Seul Nick Winter survivra à la Première Guerre Mondiale.

6 Mais bien plus, ce sont les grandes œuvres de la littérature populaire française, dont le succès n'est plus à prouver, qui intéressent les studios et constituent leur deuxième grande source d'inspiration. C'est encore Victorin-Hippolyte Jasset qui, en précurseur toujours, et toujours pour le compte de la société Éclair, réalise en 1911 Zigomar, puis Zigomar, roi des voleurs, adaptés de Zigomar, le maitre de l'invisible qui met en scène un personnage de criminel encagoulé créé par Léon Sazie, dont les aventures ont paru en 1909 et 1910 dans Le Matin (164 épisodes). Fort de l'énorme succès du film, il réalise une suite, en partie tournée à Paris, en décors naturels : Zigomar contre Nick Carter (1912) puis Zigomar, peau d'anguille (3 épisodes - 1913). Son décès, en juin de la même année, met un terme à la série. Il laisse cependant derrière lui les bases d'un modèle pour le film policier.

7 Face à la société Éclair, les deux principales sociétés de production cinématographiques de l'hexagone ne pouvaient pas ne pas réagir. Et toutes deux se tournent vers Fantômas, une série en cours de parution depuis février 1911 chez Arthème Fayard, directement en volumes (et c'est une nouveauté éditoriale) dont chacun peut se lire séparément, et qui connaît un succès sans précédent : 400000 exemplaires vendus en quelques mois, un deuxième tirage dès septembre (Marcel Allain dira même que les tirages ont dépassé ceux de la Bible!) ${ }^{5}$. Pathé propose 2000 francs or aux auteurs pour les droits. Léon Gaumont, pourtant toujours soucieux d'économie et d'ordinaire peu enclin à payer des droits d'auteur (il considère que c'est payer deux fois un même scénario), se laisse 
convaincre par Louis Feuillade. Il propose 6000 francs or, une somme importante à l'époque, et remporte la mise.

8 Le perdant, Pathé, se tournera alors vers Rocambole pour une adaptation des aventures du personnage de bandit repenti en justicier créé par Alexis Ponson du Terrail, parues pour la première fois en 1857 dans le journal La Patrie. Le premier film de cette série en 3 épisodes, réalisé par Georges Dénola, La Jeunesse de Rocambole, sortira en mars 1914, mois durant lequel sortira le quatrième film adapté de Fantômas.

9 C'est donc dans ce contexte de concurrence exacerbée entre sociétés de production cinématographiques qui recourent en série aux héros positifs comme négatifs de la littérature populaire, que Louis Feuillade, ancien journaliste polémiste, entré en 1905 comme scénariste chez Elgé ${ }^{6}$, très rapidement réalisateur à succès, puis directeur artistique de la société Gaumont (1907), qui s'est déjà essayé au genre, adapte et réalise les premiers volumes d'une série à succès toujours en cours de parution, et qui comptera en tout 32 volumes de 400 pages chacun (le dernier, la Fin de Fantômas, paraît le 20 septembre 1913, quelques jours après la sortie du deuxième film de Louis Feuillade) ${ }^{7}$.

\section{Fantômas (tout) contre Fantômas : Hypotexte, Paratexte et Filiation}

\section{A. Fantômas s'affiche}

Louis Feuillade prend soin, dès le départ, d'associer les auteurs à son travail. «Je suis enchanté de tourner Fantômas! Nous allons faire du bon travail! Vous verrez!» (Lacassin 1964 : 156). Et lorsque le 9 mai 1913, sort au Gaumont-Palace à Paris ${ }^{8}$ le premier film adapté du premier volume paru, respectant ainsi l'ordre de parution, il semble bien aux vues du matériel publicitaire que Louis Feuillade se mette dans les pas de l'œuvre originale. Bien plus, l'affiche du film qui s'étale sur tous les murs aussi bien que les brochures publicitaires semblent assumer, voire revendiquer explicitement une filiation?. 


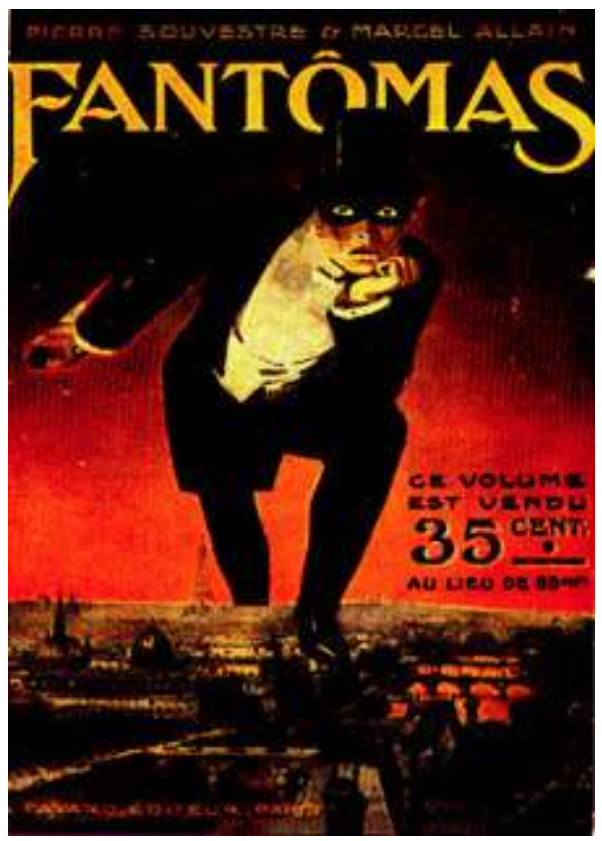

Collection privée

Affiche du film adapté du roman

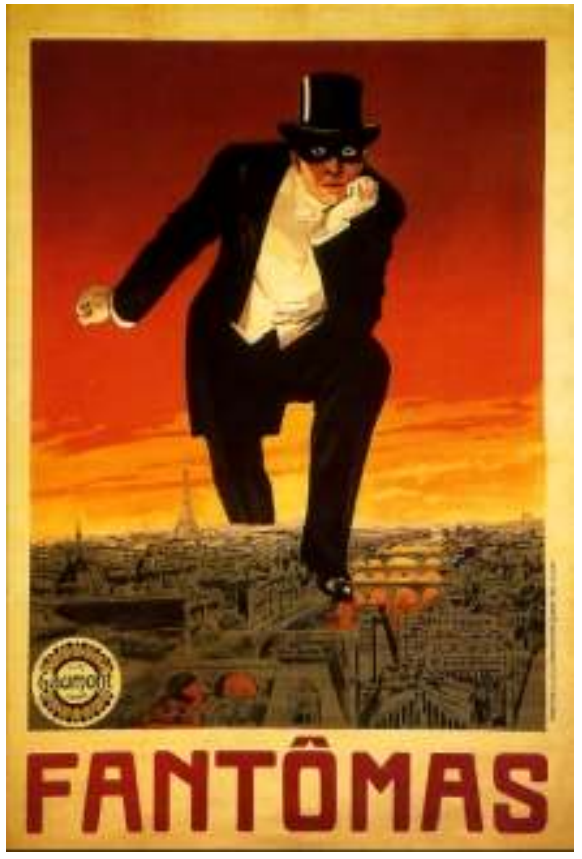

Gaumont

11 L'illustrateur-maison de l'éditeur Fayard, Gino Starace, avait illustré la première de couverture du roman en s'inspirant d'une affiche publicitaire anonyme vantant une boite de pilules (Pink) qui avait retenu l'attention de Pierre Souvestre et Marcel Allain. Il avait remplacé la boîte de pilules par un poignard sanguinolent et fait disparaître les pilules lâchées sur Paris. C'est cette couverture, et non pas, par exemple, une scène narrative du film, que l'affiche reprend. 

violence de l'image : il supprime le poignard et le sang, éclaircit sa palette, stylise les traits de Fantômas. De fait, ce dernier, dans une pose plus statique que sur la couverture originale, sa silhouette décentrée vers le haut et mise à distance par un cadre couleur crème, apparaît moins menaçant. Le titre du film (Fantômas - À l'ombre de la guillotine) est amputé au profit du seul héros éponyme. On peut y voir le même souci d'en édulcorer la violence en supprimant toute référence à la mort, à la violence, au crime (mais nous y reviendrons plus loin) - et quel formidable aveu, par là-même, du pouvoir de l'image. On peut également y voir la logique publicitaire : un titre court sur un fond clair en marge de l'illustration, c'est un titre efficace. Mais reprendre ainsi le titre même du roman, tout comme l'affiche reprend la couverture, c'est rendre hommage à l'hypotexte, réaffirmer sa filiation à l'œuvre littéraire originale. C'est aussi un argument de vente, avec toute l'efficacité commerciale que cela suppose : autrement dit, c'est placer le succès attendu, ou pour le moins espéré, dans celui du roman. Ce qui, notons-le, souligne encore la parenté au niveau des contraintes économiques et commerciales entre ces deux arts de masse que sont la littérature populaire et le cinéma.

13 La couverture du livre reste fortement reconnaissable. Il s'agit donc, avec ces aménagements de façade, mais qui concernent paradoxalement les parties les plus exposées du film (celles qui figurent sur les frontons du cinéma, dans la rue, la presse, le programme publicitaire), de renvoyer le spectateur potentiel non pas tant à un « déjà vu» (la couverture du livre), qu'à un « déjà lu ». Paradoxalement ce qui différencie vraiment la couverture de l'affiche, c'est la lettre : si l'illustration de la couverture est saturée d'information écrite, celle de l'affiche apparaît singulièrement dépouillée. Ce que ne manqua pas de noter Renée Carl, l'interprète de Lady Beltham, dans ses mémoires inédites citées par Jean-Louis Capitaine : «Sur les affiches, seule la marque de la grande firme rayonnait » (70). De fait, mis à part le titre, la marguerite ${ }^{10}$, qui accompagne toutes les éditions, quelles qu'elles soient, des produits Gaumont (des appareils et pellicules aux étiquettes et prospectus), rayonne tel un copyright. Et de créateurs, qu'ils soient auteurs de l'œuvre originale, réalisateur, adaptateur, acteurs, voire affichiste (et ce, comme le souligne J.-L. Capitaine, en contradiction avec la tradition française de l'affiche illustrée, y compris au cinéma), il n'est point fait mention, questionnant ainsi non plus le statut de l'adaptation cinématographique, mais le cinéma lui-même, où, à l'instar de la littérature populaire, les créateurs s'effaceraient derrière l'œuvre - ici l'œuvre originale.

14 Examinons de même un encart publicitaire Gaumont de quatre pages annonçant la sortie de la série de films : 


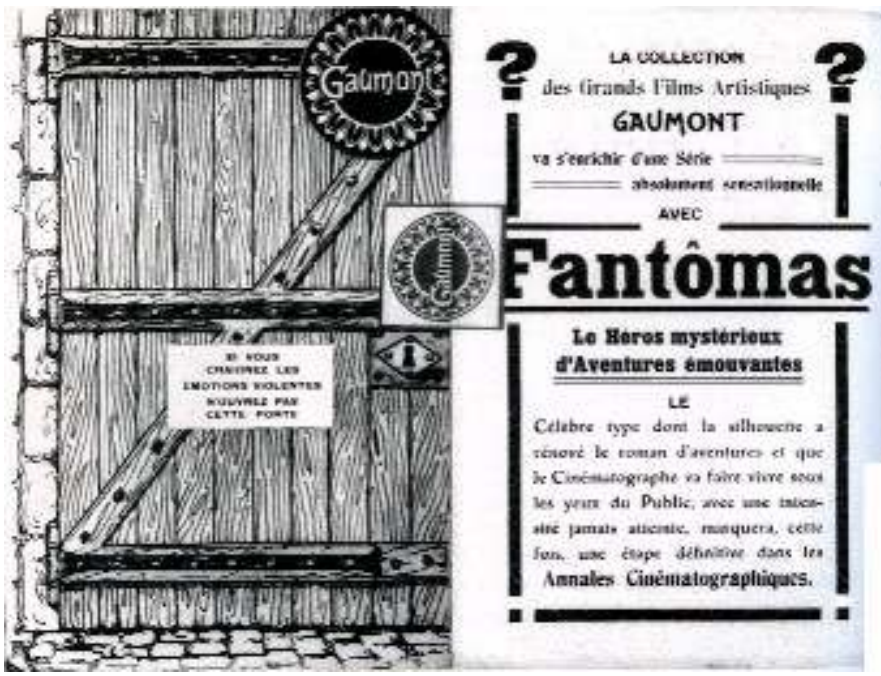

Collection privée

Encart publicitaire Gaumont 2/2
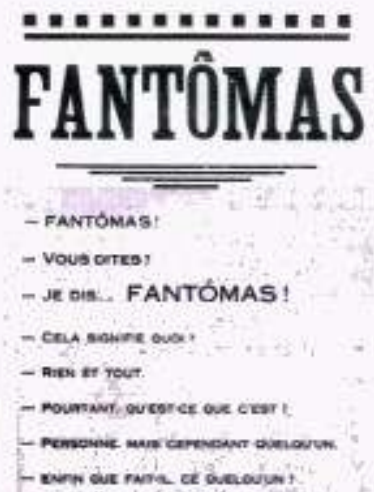

il fait PEUR.

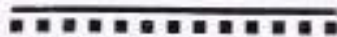

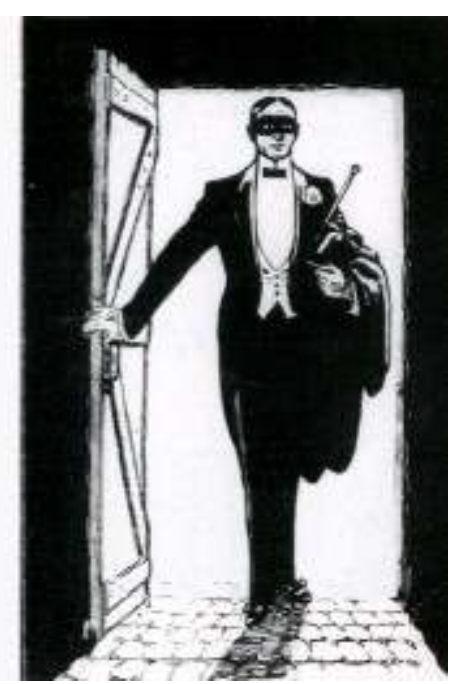

Collection privée

Ce matériel recourt à l'émotion et au mystère, associant texte, image et manipulation : le futur spectateur est invité à desceller la brochure fermée par la marguerite Gaumont, à ouvrir ainsi lui-même la lourde porte barrée du cachot («si vous craignez les émotions violentes n'ouvrez pas cette porte »); à droite, un texte, encadré de noir comme un faire-part surmonté de points d'interrogation, au vocabulaire emphatique qui n'est pas sans rappeler les ressorts de la littérature populaire («absolument sensationnelle »), inscrit le film, via son héros (et le titre éponyme cultive l'ambiguïté), dans les pas $\mathrm{du}$ «roman d'aventures». Une fois la porte ouverte, libérant ainsi Fantômas en dandy au loup noir (le costume de l'affiche), la parenté avec l'œuvre littéraire est rappelée : le fameux dialogue du roman est reproduit à gauche :

«- Fantômas!

-Vous dites?

- Je dis ... FANTÔMAS!

[etc...]» 
Cet encart publicitaire, bien plus que l'affiche, révèle la posture de Louis Feuillade face à l'œuvre originale qu'il est en charge d'adapter. D'une part, il se réclame clairement de l'hypotexte, y renvoie le spectateur comme un préalable au film grâce à des références aisément décodables et dans un style rappelant la littérature populaire, - tout en clamant la supériorité émotive du cinéma qui va «faire vivre [le célèbre type] sous les yeux du public avec une intensité jamais atteinte». D'autre part, il définit non seulement son œuvre comme cinématographique, mais la considère comme une pierre de touche "définitive " dans l'histoire du cinéma. C'est bien en adaptateur-créateur que Louis Feuillade entend œuvrer.

17 Les auteurs n'en seront d'ailleurs pas dupes: «Nous étions, Souvestre et moi, bien souvent à ses côtés. Faisions-nous donc un travail d'équipe? Sûrement pas!». D'ailleurs, les propos de Feuillade tels que Marcel Allain les rapporte ne laissent guère de doute : «[...] A vous de me donner des idées! Si elles me plaisent ${ }^{11}$, je me charge de les traduire en images... » (Lacassin $1964: 156)$.

\section{B. Fantômas défile : Tresse narrative et linéarité filmique}

Adapter au cinéma un roman tel que Fantômas, aux caractéristiques narratives inhérentes au genre (plusieurs centaines de pages, de multiples personnages, une succession d'actions, d'intrigues, des aventures croisées, des rebondissements à rallonge jusqu'à l'incohérence ou tout simplement laissés en suspens, un style exagératif, des énumérations sans fin, un vocabulaire emphatique), le tout dicté à deux voix, ce n'est pas seulement relever une gageure. C'est d'abord se heurter à la longueur de pellicule, non seulement parce que l'attention du spectateur a ses propres limites, différentes de celles du lecteur qui a, lui, loisir de fractionner sa lecture, mais aussi pour des raisons techniques qui portent tout à la fois sur la pellicule, l'équipement, l'opérateur, tout ceci entraînant des pratiques de consommation nouvelles.

19 De fait, si la norme en matière de longueur reste, à l'époque, entre 100 et 250 mètres, on atteint avec la sortie, en janvier 1913, des Misérables d'Albert Capellani (Pathé) les 3600 mètres, soit plus de 3 heures. Cet allongement des films n'entraine pas seulement une nouvelle définition de la durée du film, désormais temporelle et non plus métrique. La capacité des projecteurs n'étant pas supérieure à 600 mètres, il faut désormais deux appareils qui se relaient durant la projection, sous la responsabilité de l'opérateur. Enfin, elle entraîne une distinction entre "grands films" et "compléments de programme ", une évolution que les professionnels n'encouragent pas car elle modifie non seulement l'organisation des programmes mais également les habitudes du public qui, comme au caf' conc', entre et sort en cours de projection. C'est, comme le souligne Jean-Jacques Meusy dans son ouvrage, la grande révolution technique de ces années (1911-13) en matière cinématographique.

20 Feuillade choisit de s'inscrire dans cette révolution, la série des Fantômas constituant les films les plus longs, et de loin ${ }^{12}$ qu'il n'a jamais tournés jusqu'alors, sans pourtant dépasser 1 heure 30. Ce qui l'inscrit dans le format romanesque mais d'une manière paradoxale : l'unité narrative autonome de chaque « fort volume » est maintenue, mais l'oblige, par là-même à supprimer des pans entiers du roman (a-t-il hésité avec la formule à épisodes) ? Ces coupes entraînent une linéarisation de la narration. On est là au cœur du procédé adaptatif de Louis Feuillade qui le conduit in fine à s'émanciper de l'œuvre originale, à faire du cinéma. 
21 Cinéaste certes, Louis Feuillade, homme de lettres, homme de plume dans la première partie de sa vie, est rentré à la Gaumont pour écrire des scenarii avant de les réaliser luimême ; comme le rapportera Henri Fescourt, autre réalisateur embauché par Feuillade en 1912 et qui, lui aussi, a commencé à la Gaumont par écrire des scenarii : "Ce qui compte dans le ciné, me répondit [Feuillade], c'est le scénario. Pour la mise en scène, c'est une question de résistance morale et physique, puis de goût. Pour la photographie, il y a l'opérateur » (Gauthier et Lacassin 99).

Dans Fantômas, encouragée par une écriture à quatre mains ou plutôt à deux voix ${ }^{13}$, la trame narrative se présente, de fait, comme une «tresse narrative " formée de trois brins qui s'entrecroisent au fil du roman, pour ne plus en former qu'un seul (chapitre 29) vers un dénouement supposé qui n'en sera pas un, série romanesque oblige.

23 Le premier ${ }^{14}$ brin est constitué par l'affaire de l'assassinat de la Marquise de Langrune. Viennent s'y greffer trois brins secondaires, que l'on appellera «La folie d'Alice Rambert » (chapitre 15), "L'explosion du Lancaster » (chapitre 23), et « Le meurtre du Paris-Luchon» (chapitre 26). Le second brin est constitué de l'affaire de l'assassinat de Lord Beltham. Il occupe une position centrale dans la tresse : préexistant à l'ouverture du roman, il organise l'ensemble de l'intrigue. Le troisième brin est constitué du vol des bijoux de la Princesse Sonia Danidoff (et de la baronne Van den Rosen).

Chaque brin s'entrecroise d'un chapitre à l'autre, à la faveur de trois interventions :

1. Des éléments fortuits introduits par des procédés narratifs bien connus: par exemple, chapitre 1 , chez la marquise, sur le lieu du crime encore à venir, une interview dans le journal La Capitale (pour lequel Charles Rambert-Jérôme Fandor deviendra rédacteur, chapitre 19) fait porter la conversation sur l'affaire Beltham (et introduit l'assassin sur les lieux du crime avant même sa présence effective) ; chapitre 2, un télégramme renvoie Juve à Paris, faisant basculer la narration sur l'affaire Beltham.

2. Le passage d'une intrigue à l'autre de personnages secondaires (simples témoins ou victimes innocentes), qui complexifie le développement et brouille les frontières. Ainsi Bouzille, le chemineau du chapitre 4 interpellé dans le cadre de l'assassinat de la Marquise, que l'on retrouve aux Halles, chapitre 17, dans le cadre d'un développement narratif secondaire (la folie d'Alice Rambert), puis devant la prison de la Santé, chapitre 33, pour l'exécution capitale de l'assassin de Lord Beltham ; ou Thérèse, la petite-fille de la Marquise qui devient collaboratrice de Lady Beltham au chapitre 13. Ou encore la baronne de Vibray que l'on retrouve dans la loge de Valgrand. On peut classer dans cette même catégorie Charles qui, accusé de l'assassinat de la Marquise, se retrouve sous une fausse identité (Mademoiselle Jeanne) dans l'hôtel où a été commis le vol des bijoux de la princesse, pour se retrouver sous celle de Fandor, identité créée de toute pièce par Juve, chapitre 19. On notera que ces personnages, en passant d'une affaire à l'autre, d'un monde à l'autre, éventuellement en changeant d'identité, dédoublent Fantômas, et redoublent l'effet inquiétant et insécurisant de ce monde, ces mondes perméables au crime.

3. Enfin, contrastant avec le récit dont la complexité n'a d'égal que l'imagination toujours renouvelée des auteurs, l'enquête que mène Juve. Elle tisse, tout à la fois, le lien entre les différentes affaires, et les dénoue : elle organise la narration, la fait progresser, lui donne sa cohérence et confère à Fantômas («Personne... mais cependant quelqu'un ») ses identités, une existence unique, une réalité.

25 En effet, au niveau de(s) (l')intrigue(s) policière(s), c'est bien Juve qui, seul car incapable de le prouver, la (les) mène et la (les) résout; qui, d'indices en observations (le fragment de carte Taride, la cicatrice de Gurn), d'interrogatoires et de filatures 
(éventuellement déguisé) en raisonnements logiques (la pâleur de Valgrand sur l'échafaud), et avec l'appui de la science anthropométrique de Bertillon attribue au seul Fantômas l'ensemble des crimes commis sous des identités différentes.

Et si le roman s'ouvre sur le portrait d'une société établie de province, celles des ordres de l'Ancien Régime - un ancien magistrat, le curé de la commune et des membres de l'aristocratie - qui constate les méfaits d'un "être mystérieux et redoutable », d'« une ombre ", c'est bien à Paris, sur un tête-à-tête entre deux hommes, un policier et un criminel condamné à mort mais "vivant ", qu'il se referme provisoirement. De fait, le volume suivant à paraître s'intitulera Juve contre Fantômas.

Le tableau ci-dessous illustre cette tresse narrative :

Nature des intrigues

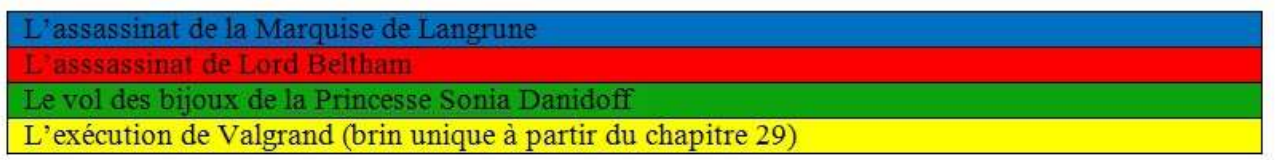

Tableau des intrigues

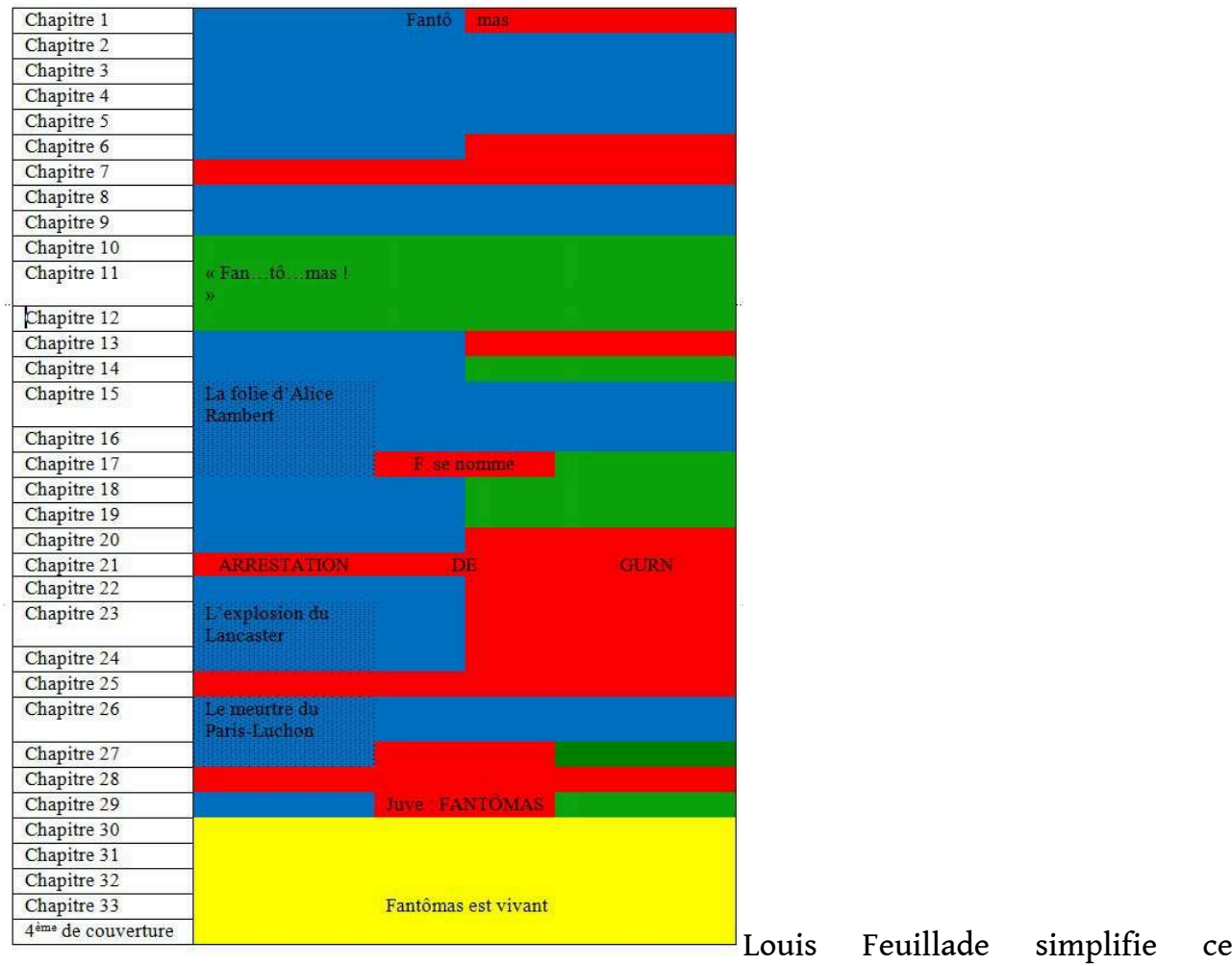

schéma narratif complexe en l'épurant, d'une part, et en lui conférant une linéarité toute filmique d'autre part.

D'abord, il dénoue les brins, en abandonne un (le meurtre de la marquise de Langrune) et tous les éléments afférents: les trois histoires secondaires, les personnages - y compris Charles -, et les identités de Fantômas qui y sont attachées - ainsi Étienne Rambert ou le professeur Swelding. Ceci a quatre conséquences principales. D'abord, le cadre se limite à des intérieurs parisiens : plus de château dans le Lot, plus de train par exemple. Ensuite, le nombre de personnages qui peuplent l'intrigue est 
considérablement diminué. De même, les personnages qui subsistent ont un profil moins complexe, Fantômas restant le seul à revêtir plusieurs identités (mais il en a perdu), plusieurs déguisements. Bien plus, la dimension mélodramatique du roman, développée autour du personnage de Charles, disparaît : son amour devenu impossible pour Thérèse qui l'oblige à renoncer à son identité (et même un temps à son sexe); sa souffrance face à l'amour que lui refuse son père (ou de celui qu'il prend pour tel) qui ne cesse de le soupçonner des différents crimes commis mais se "sacrifie " pourtant pour lui, et dont le destin restera mystérieux; la folie présumée de sa mère; la révélation impossible de son innocence. Le personnage de Fandor journaliste subsiste dans son rapport privilégié avec Juve, certes, mais en simple témoin bienveillant de l'enquête.

Puis, si Feuillade conserve les deux autres brins de la tresse romanesque (l'assassinat de Lord Beltham et le vol des bijoux, qui ouvre désormais la narration filmique), il les organise différemment, dans la linéarité. Il s'agit désormais, dans une métaphore capillaire filée, d'extension davantage que de tresse.

Conservant le procédé du télégramme utilisé par Pierre Souvestre et Marcel Allain pour envoyer Juve d'une affaire à l'autre, des indices incontestables fondent rapidement les deux affaires en une seule, autour d'un seul et même criminel, Fantômas que Juve, sur ses traces, finit par arrêter. On remarquera, avec le passage du roman au film, du texte à l'image, la part importante que joue désormais la lettre dans l'intrigue : les cartes de visite, l'initiale et l'adresse figurant dans le chapeau, les lettres échangées par Fantômas et Lady Beltham en prison, celle signée par Nibet le liant à l'évasion. La lettre vient suppléer le langage parlé, et non pas seulement dans les sous-titres.

Dans ce nouveau schéma narratif à la progression narrative linéaire épurée, le tableau devient le suivant ${ }^{15}$ : 
Tableau des intrigues

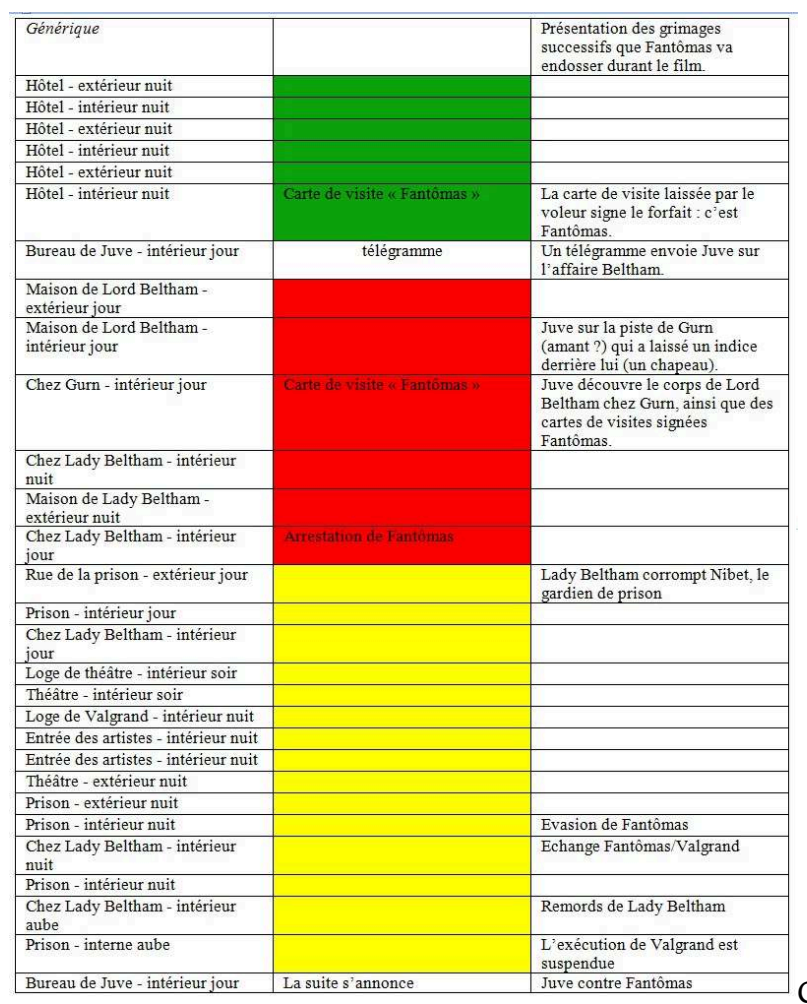

Cette linéarité du récit, acquise par le processus adaptatif, a deux conséquences principales. D'abord, elle inscrit résolument le film de Feuillade dans un genre. Finie la dimension mélodramatique largement développée autour de Charles puis laissée en suspens dans le roman. Caractéristique du déroulement de l'intrigue : un crime, un fin enquêteur à la poursuite du criminel, une piste remontée à la faveur d'indices, une arrestation, une condamnation. De fait, si dans le roman Juve cherche plutôt à prouver l'identité de Fantômas, dans le film il s'agit de le pister.

Ensuite, elle modifie la nature inquiétante de l'univers fantômassien. Dans le roman, l'inquiétude naît d'une fresque de mondes perméables et intriqués (le Lot aristocratique, le Paris de la rive droite comme de la rive gauche, le monde des Halles comme celui des transatlantiques d'Amérique du Sud), de personnages aux identités multiples (y compris l'inspecteur Juve), traversés d'une série de crimes horribles, complexes et mystérieux, où l'ombre de Fantômas rôde, plane et menace, partout, un et multiple, là sans être là puisqu'on ne peut le prouver, insaisissable puisqu'il s'échappe, incarnation du Mal et vainqueur sur le Bien, sur l'Ordre et la Justice censés protéger la société, et qu'il manipule et pervertit. N'est-ce pas la Justice elle-même (par la main de son bourreau) qui exécute un innocent sous les yeux de la police impuissante (Juve) ? Et à la fin, « Fantômas est [toujours] vivant ». Serait-il donc éternel ?

Le ressort narratif du film, lui, met en scène un Fantômas " génie du crime » qui, au fil de la pellicule, prend une dimension diabolique, violant la loi comme la morale. Du simple «voleur de visages » (Didier Blonde) tel que le générique le présente, il se mue en voleur de bijoux dans le style d'un gentleman-cambrioleur (il laisse sa carte de visite à sa victime), puis voleur d'épouse (Fantômas amoureux, le meurtre de Lord Beltham ne serait-il pas un crime passionnel?), pour enfin se muer en voleur d'identité. Manipulateur, machiavélique et sans scrupule, narguant la loi et l'ordre, il sacrifie une 
victime grotesque, innocente et inconsciente (donc triplement incapable de se défendre), pour son propre salut. À la fin, cependant, le Bien finit par s'imposer sur le Mal : l'ordre est rétabli en partie (car c'est du provisoire, série oblige) grâce à Juve qui dévoile la supercherie et sauve Valgrand de la mort. Et si Fantômas a réussi à s'échapper, c'est un face-à-face qui s'annonce désormais, face-à-face qui suppose donc égalité de statut, mais aussi deux facies.

Le dernier plan du film montre Juve, assis à son bureau, fumant d'un air méditatif. Apparait un Fantômas fantomatique (en surimpression) qui vient le narguer. De même que pour le roman, où le paratexte annonce, en $4^{\mathrm{e}}$ de couverture, le titre et la date de sortie du volume suivant, la dernière scène du film annonce le film suivant justement titré Juve contre Fantômas. Et notons également que Fantômas est ici tel que l'affiche du film le représente : imberbe, en habit, loup noir et chapeau haut de forme.

Ainsi, le film renvoie, au final, à l'œuvre littéraire, ou plus exactement à la couverture du livre, tel une invite à le rouvrir, tel un hommage. Entre l'affiche placardée dans Paris ("Sur tous les murs s'étale l'affiche du bandit masqué tenant Paris dans ses griffes » raconte René Carl (Capitaine 85)) et la scène finale du film, la boucle est bouclée.

\section{Fantômas, le masque, la plume et l'écran ou Fantômas s'émancipe}

\section{A. Pas de masque pour Fantômas (Le générique : procédé cinématographique, esthétique théâtrale et fiction littéraire)}

Adapter Fantômas au cinéma, c'est l'organiser différemment dans une durée et une linéarité proprement cinématographiques : celle de la pellicule. C'est donc faire des choix, procéder à des coupes dans la narration; mais c'est aussi confronter des procédés littéraires aux exigences d'un art visuel. Et Fantômas soulève bien des défis, à commencer par la représentation de son héros éponyme.

Comment représenter visuellement un personnage dont le nom renvoi ${ }^{16}$, de manière transparente, à la figure-oxymore du fantôme ${ }^{17}$ ?

Comment représenter au cinéma « Personne... mais cependant quelqu'un! », quelqu'un d'indéfinissable, dont le nom même est insaisissable («Vous dites?») et peu signifiant («Cela signifie quoi? »), sauf à le comparer au néant ou son contraire holistique (« Rien ... et tout!»). Bien plus, si, comme le souligne Annabel Audureau, Fantômas est un génie du crime dont «l'existence première [...] réside dans la réaction qu'il provoque dans l'émotion d'autrui » (Audureau, 53), comment donc représenter à l'écran un personnage qui se définit tout à la fois hors-champ ${ }^{18}$ et dans le regard du spectateur?

Comment représenter au temps du muet un personnage qui n'est qu'une "voix », alors que, comme le fait justement remarquer Didier Blonde, « dans le texte, l'incarnation est d'abord un pseudonyme mystificateur. On n'en voit que le nom. L'image montre, le mot cache » (Blonde 50)?

Pour résoudre toutes ces questions, Louis Feuillade, qui est monté sur les planches en amateur du temps de sa jeunesse à Lunel, qui s'est essayé à l'écriture d'un vaudeville, dont les seules sorties en dehors des parties de pêche sont pour le théâtre et dont la bibliothèque, à sa mort, comptera près de 3000 pièces de tous genres, pays et époques, se tourne vers sa source d'inspiration de toujours ${ }^{19}$. Spectacle de masse mais ancien et 
traditionnel, culturellement ancré, le théâtre, spectacle visuel, offre aux spectateurs via son esthétique, un langage connu de tous.

41 Certes, dans le roman, il est déjà fortement question de grimage, de rôles : Fantômas «l'homme aux mille visages » (Didier Blonde) -, mais aussi Juve (tour à tour chemineau, agent d'une compagnie maritime, surveillant au Royal Palace Hôtel, homme vert) ou encore Charles (Mademoiselle Jeanne puis Fandor) se déguisent et se travestissent. Feuillade a recours au même procédé théâtral, mais le réserve désormais (au moins dans ce premier film) au seul Fantômas - et à son pâle imitateur, son double de tragicomédie, Valgrand. Le travestissement change ainsi de nature: il ne s'agit plus de dissimuler Fantômas derrière des identités multiples (celles que lui confèrent ses déguisements successifs), mais paradoxalement, de le désigner puisque lui seul, désormais, se déguise.

En appui à ce dispositif et pour en assurer la lecture, Feuillade en présente le code et le décode avec le public avant la première scène du film: c'est la fonction assignée au générique qui tire partie de la technique cinématographique, - de ses limites (une prise de vue frontale offerte par une caméra encore fixe, à l'instar du point de vue du spectateur au théâtre), comme de ses innovations: le fondu enchaîné. Et pour distinguer le générique du corps du film, Feuillade fait encore appel à l'esthétique théatrale: entre théâtre de marionnettes, spectacle de pantomime et spectacle de transformation, il filme, sur un fond de toile peinte, un acteur cadré en haut du corps, muet, au jeu statique, un mouvement de tête et une direction du regard, bouche close.

En gros plan, l'acteur René Navarre, qui joue Fantômas. Il présente un visage nu, imberbe, les cheveux soigneusement tirés de part et d'autre d'une raie centrale, en costume de ville : col amidonné, veste et cravate. Son regard balaye de gauche cadre à droite cadre, en prenant soin, au passage, de regarder le spectateur dans les yeux. Fondu enchaîné : on assiste à sa transformation, sa transmutation en Fantômas-voleur de bijoux, portant cette fois barbe et moustache, animé d'un regard inquiétant qui revient vers le spectateur et le scrute. Nouveau fondu enchaîné et voici qu'apparaît Fantômas-groom d'hôtel, imberbe, regard encore plus inquiétant, de biais, droite cadre puis gauche cadre, sans vraiment s'attarder sur le spectateur. Fondu enchainé et Fantômas-Gurn apparaît, moustache noire et postiche, qui regarde le spectateur, jette un coup d'œil à gauche cadre, puis revient vers lui, comme par un jeu de fascination mutuel, avant de se fondre et disparaitre. ${ }^{20} \mathrm{Et}$ comme le remarque Paul Bleton ${ }^{21}$, ces plans de génériques, à l'entrée du film, servent en quelque sorte d'adieux au théâtre avant d'entrer dans un film qui explorera des espaces bien différents de ceux que permet la scène.

Quoiqu'il en soit, cet outil didactique offert en préalable à l'intrigue en révèle les ressorts puisqu'elle dévoile les différentes identités du criminel par ordre d'apparition à l'écran. Elle nécessite donc un contrat tacite avec le public : happé, embarqué par René Navarre qui l'invite à le suivre du regard, le spectateur accepte, de fait, d'entrer dans ce jeu de rôles, de codes et de conventions, de transformations, à l'instar de ceux qui règnent sur l'esthétique théâtrale, bien connues de lui : non seulement grimage et costumes, mais aussi adhésion à l'illusion comme réalité.

Or, sur ce point, le dispositif théâtral destiné à traduire en termes cinématographiques une réalité littéraire a-t-il parfaitement fonctionné auprès du public ? On peut se poser la question. En effet, Annabel Audureau rapporte dans son ouvrage (Audureau, 117) que René Navarre reçut des menaces de mort comme des témoignages de soutien, et 
raconte que des clients ayant reconnu Fantômas dans un café parisien en vinrent aux mains, les uns le défendant, les autres défendant une morale bafouée par cet anti-héros accusé de donner des idées aux spectateurs.

Il est vrai que René Navarre, s'étant présenté à nu à l'écran, s'est mis de lui-même en décalage par rapport à l'illusion de réalité qu'était traditionnellement sensé incarner un acteur (au moins, jusque-là, au théâtre). En effet, il s'agissait pour lui de pouvoir être reconnu par son public ${ }^{22}$, s'opposant ainsi à la politique de Léon Gaumont qui faisait tout pour limiter la notoriété de ses acteurs, par peur que ceux-ci ne réclament une hausse de leurs émoluments ${ }^{23}$. Voulant être reconnaissable, et effectivement reconnu, René Navarre, s'émancipant du système Gaumont, inaugurait ainsi le statut de vedette de cinéma ${ }^{24}$.

Ironiquement, les traditionnelles frontières entre la fiction (Fantômas) et la réalité (René Navarre) se trouvant brouillées, la question de la représentation du réel tragiquement posée dans le roman (jusqu'où le comédien Valgrand peut-il se substituer à Fantômas), trouvait un écho dans son adaptation même - et, ironiquement toujours, pour le plus grand profit de la fiction, le personnage de Fantômas se prêtant bien à cet entre-deux: ainsi, décidément, il semble que Fantômas ne soit pas " incarnable », ou qu'on ne puisse que lui prêter une réalité.

Pour finir, notons que Louis Feuillade reprendra le procédé du générique notamment dans le film suivant, Juve contre Fantômas, conférant par là-même à cette entité proprement cinématographique une autre fonction: celle d'inscrire le film dans une série, de lui assurer donc une cohérence sérielle à l'instar du roman ${ }^{25}$.

\section{B. Fantômas entre en scène : Références théâtrales, œil cinématographique}

49 À l'écran comme à la scène, et à la différence de l'écrit, les personnages s'incarnent, au sens propre du terme. Et la référence au théâtre est forcément incontournable, même si, d'ailleurs, de manière surprenante, elle n'est pas toujours assumée par Louis Feuillade. Pour preuve, ce qu'il dit de ses acteurs dans un entretien paru dans Le courrier cinématographique, le 28 mai 1914 :

50 "[J]e préfère [...] avoir mes propres acteurs. En voici une des principales raisons. Les acteurs de théatre ne sont pas libres de disposer de tout leur temps. Ils ont leurs matinées et leurs répétitions... Avec nos propres acteurs, c'est différent, nous les avons toujours sous la main... De plus, nos acteurs se passionnent pour le cinéma, et en font leur métier » (Audureau 116-117).

51 Pourtant, dans les faits, ces acteurs maison, René Navarre (Fantômas), Edmond Bréon (Juve), Renée Carl (Lady Beltham) ou encore Georges Melchior (Fandor) dont la plupart viennent du théâtre, qui se partagent souvent l'affiche et tournent quasi exclusivement avec Feuillade (René Navarre a déjà tourné avec lui plus de 80 films avant d'incarner Fantômas), ont tout d'une troupe. Or c'est bien cet effet de troupe qui, paradoxalement, confère ce jeu particulier au rythme soutenu si caractéristique des productions Gaumont, ce jeu libre et naturel, libéré de la pantomime, que l'on reconnaît aux acteurs de Feuillade. 
Danidoff telle qu'elle figure dans le roman au chapitre 10, «Le Bain de la Princesse Sonia ». Disparu le climat érotique entretenu par la perversité courtoise de Fantômas où le vol frise le viol. Ici, la scène se déroule dans la chambre et si Fantômas terrifie la princesse c'est d'abord par sa présence inopinée et la menace qu'il représente pour ses biens, non pour sa vertu. Or, dans cette scène d'intérieur réinventée, saturée de tapis, tentures, boiseries, lit, divan, meuble secrétaire et qui inaugure, dans le film, l'apparition de Fantômas, la référence au théâtre s'impose. Derrière les tentures, telles des pendrillons masquant les coulisses, attendant son entrée en scène, se cache Fantômas. Pourtant, c'est à gauche-cadre qu'il surgit, côté jardin : Feuillade convoque ainsi les codes de l'esthétique théâtrale mais les réinterprète à son profit : le criminel est traité en héros ; du côté positif émanent le danger, la menace. Feuillade fait appel au théâtre certes, mais pour mieux faire du cinéma. Et une nouvelle fois, ce brouillage des codes esthétiques traditionnels, rendant encore plus inquiétant l'univers fantômassien, est au service de l'œuvre elle-même.

\section{Anastasie défie Fantômas : cinéma, contexte, (auto)censure}

56 Au-delà des questions d'esthétique, le film, avec le personnage de Valgrand et le destin que lui réserve l'adaptation cinématographique, propose différents angles pour aborder 
la question des rapports entre théâtre et cinéma qui se pose déjà, malgré sa jeunesse, et notamment du fait de son succès auprès des foules, en rival.

À l'instar du roman, il est fortement question de théâtre dans le film avec ce portrait de comédien réduit à la caricature, que le public regarde se grimer, s'admirer et cabotiner devant ses admirateurs/trices, puis succomber à la tentation de la séduction, pour être ensuite substitué à Fantômas et échapper de justesse à l'échafaud, en pauvre imitateur. C'est la grande transformation narrative par rapport à l'œuvre originale (dans laquelle il est guillotiné en lieu et place de Fantômas), et qui donc, à ce titre, pose question.

Ainsi, Christophe Gauthier commente comme suit la scène de Valgrand se maquillant dans sa loge: «On peut lire cette visible métamorphose comme une métaphore du cinéma : quand le théâtre [...] nous dévoile et imite par des artifices connus de tous, ici le maquillage, la fausse moustache, la réalité, quand le théâtre rend le drame comique [...], le cinéma, lui ne cède rien de son mystère » (2008: [36:13-36:45]). Il n'y voit pas «meilleure démonstration [...] de la puissance du cinéma où le théâtre échoue mais où le personnage de cinéma lui parvient toujours au fond à s'évader » (2008 : [37:23-37 : 33]).

59 Annabel Audureau, elle, y voit d'abord l'expression de la sensibilité « royaliste du catholique et conservateur directeur artistique de la 'Gaumont'« (109) ainsi qu'« une forme de respect symbolique du cinéma, encore jeune, qui se refuse à évacuer si vite son aïeul théâtral » (110), la fin constituant « une métaphore même du cinéma dont le paradoxe réside dans la mimésis la plus absolue et, pour le spectateur, la simultanéité de perception entre le réel et l'imaginaire. S'il existe bien un art des métamorphoses du réel et du rêve : il s'agit du cinéma » (110).

On pourrait également avancer que l'acteur René Navarre réussit à incarner Fantômas (d'ailleurs quatre films suivront) tandis que le comédien (Valgrand) y échoue (il est démasqué).

61 Mais ces interprétations, pour justes et séduisantes d'un point de vue esthétique qu'elles puissent être, ignorent cependant une réalité proprement cinématographique et commerciale (celle du divertissement de masse) : la censure. Une censure dictée par le contexte: un environnement social et politique qui ne vise pas tant l'expression cinématographique que le media lui-même, car comme le note Christophe Fouassier: « le cinématographe entretiendrait, plus que toute autre forme artistique, un rapport ambigu avec le crime ». Et Feuillade avec son film au héros, au sujet et au titre si évocateurs, sont en première ligne.

62 Le 11 janvier 1909, après trois ans ${ }^{26}$ d'interruption durant lesquelles le débat pour l'abolition de la peine de mort a fait rage dans un contexte de crise sécuritaire hérité du $\mathrm{XIX}^{\mathrm{e}}$ siècle ${ }^{27}$, et marqués par une » affaire Soleilland » ayant vivement ému l'opinion, avec le relai efficace de la presse populaire ${ }^{28}$, les exécutions capitales avaient repris : quatre membres de la bande Pollet qui avaient commis de nombreux vols et une série de meurtres entre 1905 et 1906 dans la région d'Hazebrouck, sont exécutés à Béthune sous les yeux de 10000 spectateurs, dans une ambiance exaltée. Pathé, qui s'est spécialisé dans les reconstitutions d'exécutions capitales dont le public est friand (on citera ainsi Histoire d'un crime dont la vue $n^{\circ} 6$, Devant la Guillotine, n'est pas sans rappeler le titre de ce premier Fantômas) ${ }^{29}$, réussit à filmer l'exécution, moins coûteuse qu'une reconstitution en studio, pour la proposer dans le cadre de ses actualités cinématographiques, sous le titre : «La quadruple exécution capitale de Béthune ». En 
réaction, le ministre de l'Intérieur diffuse, à l'adresse des préfets une circulaire rappelant que si «[1]e maire a tous pouvoirs [...] pour exercer censure préalable», « il est indispensable d'interdire radicalement tous spectacles cinématographiques publics de ce genre, susceptibles de provoquer des manifestations troublant l'ordre et la tranquillité publics $»^{30}$.

Bien que le premier texte normatif de portée nationale spécifiquement consacré à l'activité cinématographique soit un arrêté du ministre de l'intérieur pris ultérieurement (le 16 juin 1916) et instituant une commission chargée de l'examen et du contrôle des films, Albert Montagne considère dans son ouvrage sur les interdits cinématographiques que cette circulaire de 1909 marque bien la naissance de la censure cinématographique en France (2007 : 23).

Christophe Fouassier, lui, fait remarquer que ce texte érige des principes généraux destinés à inspirer les pouvoirs publics au-delà de la seule affaire de Béthune et que c'est une lecture extensive de la notion de "spectacles cinématographiques de ce genre » qui a prévalu, insistant davantage sur le risque de troubles que sur la représentation des exécutions capitales proprement dite. Et de fait, sous la forte pression de leurs administrés ou par simple souci électoral, les maires prennent nombres d'interdictions municipales visant la représentation à l'écran de simples actes criminels, pour des motifs de plus en plus divers et selon un arbitraire tout local (61), provoquant les critiques du Syndicat des Directeurs de Cinématographie. Cela donne lieu à une nouvelle circulaire, le 19 avril 1913, à trois semaines de la sortie de Fantômas, à l'ombre de la guillotine. Cette circulaire confirme l'interdiction de représenter des exécutions capitales mais aussi désormais « les représentations de crimes récents par le cinématographe ", et renforce les pouvoirs du préfet en matière d'interdiction: la censure s'uniformise, s'organise au niveau national; de morale (locale) elle devient politique (l'État). Et si la circulaire ne s'intéresse qu'aux films s'inspirant de faits réels, sans inclure les films de pure fiction ${ }^{31}$, dans les faits, et comme pour celle de 1909, les interdictions toucheront aussi les films dénués de tout lien avec l'actualité criminelle.

Ainsi, dans ce contexte, l'originalité narrative que constitue, dans l'adaptation cinématographique de Fantômas, la grâce de Valgrand, ne s'interpréterait pas tant d'un point de vue esthétique, même s'il éloigne définitivement d'une esthétique sanguinolente à la Grand Guignol, mais plutôt dans une logique de censure en interne, dans le but d'éviter tout risque d'interdiction administrative et de pertes financières, pour le producteur comme pour l'exploitant, comme n'importe quelle entreprise commerciale. On y relierait alors l'affiche édulcorée et le titre amputé du film.

Quoiqu'il en soit, et comme le fait remarquer Francis Lacassin dans sa contre-histoire du cinéma, le conformisme, l'«inébranlable confiance dans les décrets de la providence, la foi dans l'ordre établi et le sentiment que les bons seront récompensés et les méchants punis " (Lacassin 1994: 47) de Feuillade et qu'il partageait avec Léon Gaumont, s'accordent finalement bien à la censure, de même qu'avec les préceptes qu'énoncera Marcel Allain dans son Mémoire sur le roman populaire à l'intention de la librairie Fayard: "S'adressant à un public divers, le roman populaire aura souci, tout d'abord, de ne choquer aucun des éléments constitutifs de ce public. Il ignorera toute question de politique, toute discussion religieuse: ce serait restreindre, a priori, le nombre de lecteurs éventuels. Il évitera - et c'est le plus difficile - tout ce qui pourra donner matière à critique, en quoi que ce soit » $(1988: 1241)$. 
67 Et Feuillade va au-delà de la simple non-représentation de l'exécution capitale : il édulcore également le personnage de Lady Beltham (non plus blonde comme dans le roman, mais brune, pour respecter les canons esthétiques du cinéma de l'époque), amoureuse éperdue, qui éprouve des remords à l'écran concernant Valgrand.

De fait, le film rencontre un grand succès... populaire: Le Petit Journal du 16 mai annonce le chiffre de 80000 spectateurs dans la semaine. Le succès de l'affiche fut aussi grand que celui du film. Et ce sera d'ailleurs la seule adaptation cinématographique de l'œuvre qui trouvera grâce aux yeux de Marcel Allain, reconnaissant même en Louis Feuillade le « parfait talent ».

\section{Feuillade fait son cinéma}

Au-delà de la question de la censure en ce qu'elle nuit à la liberté de création et d'expression du créateur, et de la question de sa légitimité, en obtempérant, de fait, à la circulaire de 1913, qui conforte la précédente ${ }^{32}$, Feuillade entérine la définition/ qualification juridique du cinéma qui y est opérée, ce qui n'est pas neutre: il se positionne, de fait, dans le débat sur la nature (juridique) du cinéma et qui l'anime depuis sa naissance : si le cinéma rentre «incontestablement » dans la catégorie des spectacles de curiosités, alors ce n'est pas du théâtre.

Le droit ne reconnaît, en effet, alors que deux types de spectacles publics : le théâtre d'une part qui, depuis avril 1906 n'est plus soumis à la censure - non pas qu'elle ait été officiellement abolie par une loi, mais parce qu'elle ne dispose plus de budget; les «spectacles de curiosités » d'autre part, définis par l'article 6 du décret du 6 janvier 1864 relatif à la liberté de l'industrie théâtrale soumis à censure municipale, sous l'œil de plus en plus vigilant de l'État. À sa naissance, le cinéma a été assimilé (« du même genre que ») à cette deuxième catégorie qui regroupe les "spectacles de curiosités, de marionnettes, les cafés dits cafés-chantants, cafés-concerts » (décret de 1864). Or, tel qu'il a évolué, la pertinence en est de plus en plus contestable - et contestée -, ouvrant ainsi, notamment avec la circulaire de 1909 et la jurisprudence abondante qui va en découler, un débat animé et fort intéressant sur ce qui distingue le théâtre du cinéma. De fait, les réalisateurs se réclament désormais d'une démarche esthétique, se revendiquant ainsi de la même « noblesse » que celle du théâtre. D’ailleurs, Fantômas ne fait-il pas partie de la collection des « grands films artistiques » de la Gaumont ?33

De même, il est vrai que le cinéma est désormais difficilement assimilable à une activité foraine : il s'est sédentarisé et l'ouverture en octobre 1911 du Gaumont-Palace, le plus grand cinéma du monde à l'époque, 3400 places, où Fantômas, production-maison, sortira à grand renfort de publicité, est là pour l'illustrer.

De même encore, on ne peut plus parler de "curiosité » du public à l'égard du cinématographe, au départ fasciné par ce procédé technique qui permet de reproduire fidèlement la réalité (une sortie d'usine ou l'arrivée d'un train en gare). On ne vient plus tant s'étonner, s'amuser ou se faire peur avec un procédé technique : avec la durée des films qui s'accroît, l'intérêt du public s'est déplacé. C'est le contenu qui intéresse car le cinéma est désormais à même de raconter une histoire. Et c'est bien le propos de Louis Feuillade avec l'adaptation, à l'écran, de ce roman de plusieurs centaines de pages. 
74 Or, alors que « les ambitions de la création cinématographique s'apparentent donc de plus en plus à celles de la création littéraire ou théâtrale » (Fouassier, 47), qu'un débat s'organise sur la question, Louis Feuillade, lui, répond à sa manière : il choisit, très certainement, la logique commerciale, celle du divertissement de masse que le cinéma est déjà devenu, remettant la question artistique à plus tard, mais apportant de facto une réponse: le cinéma n'est pas du théâtre. Il formulera d'ailleurs sa réponse en novembre 1922, dans un entretien pour Les Films chez soi, déclarant à Marcel Allain : " On ne fait pas courir des moutons sur un champ de courses !... Pourquoi demander de faire du ciné à ceux qui ne connaissent point l'écran? D'ailleurs, c'est très simple: passez les grands succès en revue. Vous trouverez toujours qu'ils sont dus à des auteurs qui s'occupent du cinéma, depuis l'apparition de cet art nouveau. Ils s'y font fait l'œil. Ils se sont assoupli l'esprit aux procédés dont l'écran dispose. Ils connaissent les effets à obtenir et ceux qui ne 'rendent' pas. » (Gauthier Lacassin 37).

75 Le cinéma est bien un art singulier, original. Entré de plein pied dans la société et les débats qui l'agitent, il est déjà un art de masse.

\section{Fantômas - À l'ombre de la guillotine ou Quand le cinéma s'émancipe...}

76 L'adaptation cinématographique de l'œuvre de Pierre Souvestre et Marcel Allain par Louis Feuillade confronte deux arts de masse : littérature populaire et jeune cinéma. Aux vues de son succès phénoménal et immédiat, et au-delà de sa réussite artistique que confirme encore aujourd'hui l'édition de la série en DVD, on peut faire de Fantômas - À l'ombre de la Guillotine (1913) le témoin du passage de relais entre la littérature populaire et le cinéma en tant que divertissement de masse.

77 Mais peut-on parler d'adaptation, alors que le langage cinématographique est lui-même en train de s'inventer? Trois principaux traits caractérisent le travail de Louis Feuillade :

78 - Confronté aux réalités cinématographiques qui s'imposent à lui, Feuillade les résout en s'émancipant de la littérature, en trouvant des solutions auprès du théâtre, participant ainsi, et sans forcément le maîtriser encore (à l'instar de ses acteurs), à l'invention d'un langage proprement cinématographique, donnant ainsi naissance à une œuvre originale.

79 - Le poids de la censure, ou tout le moins sa menace a, paradoxalement, participé à cette double émancipation, la contrainte encourageant ici la créativité.

80 - Mais Feuillade n'en a pas moins brandi l'ombre tutélaire et protectrice de l'œuvre originale, et de la manière la plus emblématique qui soit, avec l'affiche du film. Comment, alors, interpréter cet adossement à l'œuvre littéraire? S'agit-il de la revendiquer pour mieux s'en affranchir, comme le montrera l'émancipation toujours croissante au fil des adaptations successives ? S'agit-il d'endosser, du même coup, le succès de la série ? S'agit-il de donner à son œuvre cinématographique une légitimité artistique, celle de la littérature (populaire)?

81 Reprenant l'affirmation d'Annabel Audureau selon laquelle «Fantômas n'est pas un personnage, c'est un concept » (42) et en se plaçant dans une logique purement sérielle, le film ne pourrait-il pas alors s'envisager non pas tant comme une adaptation réussie de 
l'œuvre littéraire, mais, et on pense à l'ultime scène du film où s'affiche la silhouette emblématique de Fantômas, comme son produit dérivé?

\section{BIBLIOGRAPHIE}

Alfu, L’Encyclopédie de « Fantômas », étude sur un classique, Amiens : Encrage Éditions, [1981] 2011.

Aimone Isabelle, « Une séance au Gaumont Palace en 1913 », in Cahiers de la cinémathèque nº3/64, décembre 1995.

Allain Marcel, Mémoire sur le roman populaire à l'intention de la librairie Fayard, rendu le 5 novembre 1938, consultable dans sa totalité dans Fantômas, tome II. Paris : Robert Laffont, Bouquins, 1988.

Audureau Annabel, Fantômas, Un mythe moderne au croisement des arts. Rennes : PUR, 2010.

Bleton Paul, S/Z, « Les impressions du Zigomar de Léon Sazie », article paru dans ce dossier.

Blonde Didier, Les voleurs de visages, Sur quelques cas troublants de changements d'identité : Rocambole, Arsène Lupin, Fantômas \& Cie. Paris : Métailié, 1992.

Chlastacz Michel, « Les trains de Fantômas ", in La vie du rail et des transports n 2457, 10 août 1994.

Capitaine Jean-Louis, Les premières feuilles de la marguerite, affiches Gaumont 1905-1914. Paris : Gallimard, 1994.

Champreux Jacques et Carou Alain (sous la dir.), Louis Feuillade. 1895, Revue de l'association française de recherche sur l'histoire du cinéma, $n^{\circ} \mathrm{HS}$, octobre 2000.

Deslandes Jacques et Richard Jacques, Histoire comparée du cinéma, tome second : « Du cinématographe au cinéma 1896-1906 ». Tournai : Casterman, 1966.

Fescourt Henri, La foi et les montagnes ou Le septième art au passé. Paris : Publications PhotoCinéma Paul Montel, 1959.

Segal Annie et Segal Abraham : « Fantômas, découpage intégral après montage », in L'AvantScène, Spécial Feuillade Fantômas, nº 271/272, 1-15 juillet 1981.

Fouassier Christophe, Le droit de la création cinématographique en France. Paris : L'Harmattan, Logiques Juridiques, 2004.

Gaudreault André : Du littéraire au filmique. Paris/Québec : Armand Colin/Nota bene, 1999.

Gauthier Christophe, La passion du cinéma : cinéphiles, ciné-clubs et salles spécialisées à Paris de 1920 à 1929. Paris : École nationale des Chartes, Mémoires et Documents nº 56, 1999.

Gauthier Christophe : Fantômas, le feuilleton cinématographique en cinq épisodes de Louis Feuillade. Les Cours de cinéma du Forum des images [En ligne], 9 avril 2008, consulté le 6 juillet 2011. URL: http://films7.com/videos/cours-de-cinema-christophe-gauthier-analyse-fantomasde-louis-feuillade 
Gauthier, Patrice et Lacassin Francis, Louis Feuillade, maître du cinéma populaire. Paris :

Gallimard, Découvertes, Art, 2006.

Lacassin Francis, Louis Feuillade. Paris : Seghers, Cinéma d'aujourd'hui, 1964.

Lacassin Francis, Pour une contre-histoire du cinéma. Paris : Institut Louis Lumière/Actes Sud, [1972] 1994.

Lacassin Francis, Louis Feuillade, le maître des lions et des vampires. Paris : Bordas et fils, 1995.

Masson Alain, Le récit au cinéma. Paris : Cahiers du cinéma, Essais, 1994.

Meusy Jean-Jacques, Paris Palaces ou le temps des cinémas (1894-1918). Paris : CNRS Editions, 1995.

Montagne Albert : Histoire Juridique des Interdits cinématographiques en France (1909-2001).

Paris : L'Harmattan, Champs Visuels, 2007.

Montagne Albert : «Crimes, faits divers, cinématographe et premiers interdits français en 1899 et 1909 », in Criminocorpus, revue hypermédia [En ligne], Crimes et criminels au cinéma, 1. Thèmes et figures, mis en ligne le $1^{\mathrm{er}}$ janvier 2007, consulté le 6 juillet 2011. URL : http://

criminocorpus.revues.org/207

Montheilet Véronique, «Fantômas à l'ombre des surréalistes » in Béhar Henri : Le cinéma des surréalistes. Paris : L'âge de l'homme, Mélusine, Cahiers du centre de recherche sur le surréalisme, $\mathrm{n}^{\circ} 24,2004$.

Séguin Laurent, Les collections de romans populaires et leur conservation dans les fonds patrimoniaux de la Bibliothèque nationale de France. L'exemple du « Livre Populaire » de la librairie Arthème Fayard. Volume 1. Mémoire d'étude sous la direction de Frédéric Barbier [En ligne]. ENSSIB, janvier 2005, consulté le 6 juillet 2011. URL : http://enssibal.enssib.fr/ bibliotheque/documents/dcb/seguin-vol1.pdf

Souvestre Pierre et Allain Marcel : Fantômas volume 1. Paris : Robert Laffont, 1961.

Filmographie

Fantômas, DVD 2 volumes, Coffret Prestige Gaumont, 12 février 2000.

Revue

Le Rocambole : «Fantômas centenaire », collectif, Amiens : Le Rocambole, n 54, printemps 2011.

Site Web

www.premiumwanadoo.com/fantomasfr/EnCours.php, consulté le 26 juillet 2011.

\section{ANNEXES}

Feuillade commence par adapter le premier volume, Fantômas (publié le 10 février 1911, mais écrit après le volume 3), puis les deux suivants, Juve contre Fantômas et Le mort qui tue, mais il s'affranchit ensuite. On remarque également, à partir de la troisième adaptation, autre trait d'émancipation, que les titres qu'il donne à ses films ne reprennent plus ceux des romans. Pour le reste, les contraintes de production caractérisées par une attention particulière portée aux réalités commerciales et financières, la nécessité d'une cadence de productivité intense, sont autant celles de la littérature populaire que du cinéma. 
Tableau des adaptations

\begin{tabular}{|l|l|l|l|l|}
\hline \multicolumn{1}{|c|}{ TITRE } & $\begin{array}{c}\text { DATE DE } \\
\text { PARUTION } \\
\text { DE L'GUVRE } \\
\text { ORIGINALE }\end{array}$ & $\begin{array}{l}\text { ORDRE DE } \\
\text { PARUTION }\end{array}$ & $\begin{array}{l}\text { DATE DE SORTIE } \\
\text { DU FILM ADAPTÉ }\end{array}$ & $\begin{array}{c}\text { ORDRE DE } \\
\text { SORTIE DU FILM }\end{array}$ \\
\hline Fantômas & 10 février 1911 & 1 & $\begin{array}{l}\text { 9 mai 1913 au } \\
\text { Gaumont-Palace à } \\
\text { Paris }\end{array}$ & 1 \\
\hline $\begin{array}{l}\text { Juve contre } \\
\text { Fantômas }\end{array}$ & $1^{\text {er }}$ mars 1911 & 2 & $\begin{array}{l}12 \text { septembre } 1913 \text { au } \\
\text { Gaumont-Palace à } \\
\text { Paris }\end{array}$ & 2 \\
\hline $\begin{array}{l}\text { Le mort qui } \\
\text { tue }\end{array}$ & 20 avril 1911 & 3 & $\begin{array}{l}28 \text { novembre } 1913 \text { au } \\
\text { Gaumont-Palace à } \\
\text { Paris }\end{array}$ & 3 \\
\hline $\begin{array}{l}\text { Le policier } \\
\text { apache }\end{array}$ & 20 juillet 1911 & 6 & $\begin{array}{l}\text { le 13 mars 1914 au } \\
\text { Gaumont-Palace à } \\
\text { Paris }\end{array}$ & 4 \\
\hline $\begin{array}{l}\text { Le Magistrat } \\
\text { cambrioleur }\end{array}$ & $\begin{array}{l}17 \text { janvier } \\
1912\end{array}$ & 12 & $\begin{array}{l}\text { le } 8 \text { mai } 1914 \text { au } \\
\text { Gaumont-Palace à } \\
\text { Paris }\end{array}$ & 5 \\
\hline
\end{tabular}

Enfin, dernière remarque, on notera qu'il s'autorise toute liberté d'équivalence entre longueur du roman et longueur du film : ainsi, le film le plus long, adapté du Mort qui tue, sa troisième adaptation, n'est pas issu du roman le plus épais, Juve contre Fantômas, adapté précédemment. Deux romans donnent naissance à une durée de film identique (59 mn), alors que l'un a cent pages de plus de l'autre. De même, on constate une tendance à l'allongement de la durée des films, le premier film adapté étant le plus court.

Durée des films

\begin{tabular}{|c|c|c|c|c|c|c|}
\hline $\begin{array}{l}\text { TITRE DU } \\
\text { ROMAN }\end{array}$ & $\begin{array}{l}\text { NBRE } \\
\text { PAGES }\end{array}$ & $\begin{array}{c}\text { PAR ORDRE } \\
\text { CROISSANT } \\
\text { DE } \\
\text { LONGUEUR } \\
\text { DU TEXTE } \\
\end{array}$ & $\begin{array}{l}\text { DATE DE } \\
\text { SORTIE } \\
\text { DU FILM } \\
\text { ADAPTÉ }\end{array}$ & $\begin{array}{c}\text { Duré } \\
\mathbf{e}\end{array}$ & $\underset{34}{\text { Métrage* }}$ & $\begin{array}{c}\text { PAR ORDRE } \\
\text { CROISSANT } \\
\text { DE } \\
\text { LONGUEUR } \\
\text { DU FILM } \\
\end{array}$ \\
\hline $\begin{array}{l}\text { Fantômas - } \\
n^{\circ} 1\end{array}$ & 414 & 4 & $\begin{array}{l}9 \text { mai } 1913 \text { au } \\
\text { Gaumont-Palace à } \\
\text { Paris }\end{array}$ & $54 \mathrm{mn}$ & $1146 \mathrm{~m}$ & 1 \\
\hline $\begin{array}{l}\text { Juve contre } \\
\text { Fantômas - } \\
\mathrm{n}^{\circ} 2\end{array}$ & 415 & 5 & $\begin{array}{l}12 \text { septembre } 1913 \\
\text { au Gaumont- } \\
\text { Palace à Paris }\end{array}$ & $59 \mathrm{mn}$ & $1100 \mathrm{~m}$ & 2 \\
\hline $\begin{array}{l}\text { Le mort qui } \\
\text { tue }-n^{\circ} 3\end{array}$ & $352^{*}$ & 2 & $\begin{array}{l}28 \text { novembre } 1913 \\
\text { au Gaumont- } \\
\text { Palace à Paris }\end{array}$ & $90 \mathrm{mn}$ & $1740 \mathrm{~m}$ & 5 \\
\hline $\begin{array}{l}\text { Le policier } \\
\text { apache }-n^{\circ} 6\end{array}$ & 313 & 1 & $\begin{array}{l}\text { le } 13 \text { mars } 1914 \text { au } \\
\text { Gaumont-Palace à } \\
\text { Paris }\end{array}$ & $59 \mathrm{mn}$ & $1120 \mathrm{~m}$ & 3 \\
\hline $\begin{array}{l}\text { Le Magistrat } \\
\text { cambrioleur - } \\
n^{\circ} 12\end{array}$ & 383 & 3 & $\begin{array}{l}\text { le } 8 \text { mai } 1914 \text { au } \\
\text { Gaumont-Palace à } \\
\text { Paris }\end{array}$ & $70 \mathrm{mn}$ & $1370 \mathrm{~m}$ & 4 \\
\hline
\end{tabular}




\section{NOTES}

1. Le cinéma de fiction est né d'abord, on le rappelle, pour promouvoir le matériel cinématographique.

2. Roman bon marché (vendu 10 cents) qui, de définir un format, finit par définir un genre littéraire.

3. Nick Carter est apparu pour la première fois dans un dime novel écrit par John R. Coryell tiré d'une histoire écrite par Ormond G. Smith The Old Detective's Pupil ; or, The Mysterious Crime of Madison Square paru dans le New York Weekly daté 18 September 1886.

4. On citera : Le Proscrit (1912), L'Oubliette (1912), La Course aux Millions (1912), Le Guet-apens (1913).

5. Le tirage cumulé revendiqué par la librairie Fayard dans les années 1950 pour l'ensemble des titres, est de cinq millions d'exemplaire.

6. Pour L.G., les initiales de Léon Gaumont.

7. Pierre Souvestre décèdera prématurément le 26 février 1914, avant la sortie du dernier film de la série. Marcel Allain reprendra ensuite seul la série pour onze volumes supplémentaires (1926-1963).

8. Le plus grand cinéma du monde à l'époque, avec ses 3400 places. Il a été inauguré le 11 octobre 1911, année de la parution des quatre premiers volumes de la série des Fantômas qui seront adaptés à l'écran (en tout, ce sont onze volumes de la série qui paraissent en 1911).

9. Les reproductions de l'affiche, ainsi que celles de l'encart publicitaire figurent sur le site dédié à Fantômas : http://www.premiumwanadoo.com/fantomasfr/

10. La marguerite (en l'honneur de la mère de Léon Gaumont dont elle rappelle le prénom) figure ici dans sa version définitive.

11. Souligné par l'auteur.

12. L'Agonie de Byzance, sa super-production sortie en juillet 1913 ne fait ainsi « que " 868 mètres.

13. Par souci de productivité et de rapidité, auxquelles ils étaient liés par contrat, les deux auteurs dictaient.

14. L'ordre de l'énumération est une simple commodité. On pourrait en effet le contester, l'affaire Beltham étant l'affaire centrale, d'ailleurs évoquée dès la $4^{\mathrm{e}}$ page du roman, avant même l'assassinat de la Marquise de Langrune.

15. Le découpage qui a été retenu est celui proposé par L'Avant-Scène du Cinéma, spécial Feuillade Fantômas.

16. Le Fantôme fut d'ailleurs le premier nom donné au personnage de Fantômas, dans ses premières aventures, L'Empreinte, qui prendront ensuite la troisième place dans la série.

17. Voir http://www.cnrtl.fr/etymologie/fant\%C3\%B4me consulté le 22 avril 2011 : 1. «image

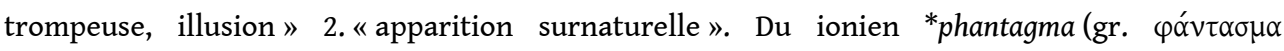
« apparition, vision ; image offerte à l'esprit par un objet ; spectre fantôme » transcrit dans le lat. impérial phantasma).

18. On notera que dans le roman, Fantômas énonciateur n'assume jamais son identité : il ne la décline qu'à la troisième personne, par le biais d'une carte de visite, ou dans le noir : « Fantômas te l'interdit!» (sic).

19. Ainsi Francis Lacassin établit le parallèle pour «La vie drôle »: «Les arcanes du vaudeville, celui de Feydeau et de Courteline, n'ont pas de secret pour lui. Feuillade en use largement dans ses scénarios pour la série "La vie drôle" " (Gauthier 2006 : 50).

20. On remarque que ne figure pas dans ce défilé inaugural le déguisement de Fantômas tel qu'il apparaît dans la dernière scène du film, preuve de sa fonction particulière.

21. Entretien (mai 2010).

22. Dans un entretien avec Annabel Audureau en septembre 2006, Jacques Champreux révélait que c'était René Navarre lui-même qui avait souhaité pouvoir être reconnu par le public. 
23. Les affichistes et dessinateurs avaient ainsi pour consigne de représenter les personnages de telle sorte qu'on ne puisse les identifier aux acteurs.

24. Il finira par quitter la Gaumont pendant la Première Guerre Mondiale pour monter sa propre maison de production : Les films René Navarre.

25. Mais cette fois, Juve, désormais l'égal de Fantômas, figure de même dans le générique.

26. En 1906, les partisans de l'abolition de la peine de mort se mobilisent: le Président de la République, Armand Fallières, grâcie systématiquement tous les condamnés à mort en sa première année de septennat; la Commission du budget de la Chambre des députés vote la suppression des crédits destinés à l'entretien de la guillotine et à la rémunération du bourreau, entravant ainsi la procédure d'exécution des condamnés; un projet de loi abolitionniste est déposé à la chambre par le Garde des Sceaux.

27. En 1907, la Société générale des prisons estime que «la criminalité augmente »: « le nombre de crimes de sang s'est élevé de 715 (1901) à 1075 (1905).

28. Soleilland, accusé d'avoir violé et assassiné une petite fille est condamné à mort, puis grâcié par le Président Fallières. Le quotidien anti-abolitionniste Le Petit Parisien, « le plus fort tirage des journaux du monde entier ", lance alors un "référendum » sur la peine de mort, accompagné d'un « concours artistique » qui reçoit 1.412347 réponses (92 \% en faveur du maintien de la peine de mort); on peut lire les articles du Petit Parisien à: http://gallica.bnf.fr/ark:/12148/ bpt6k562416v.

29. Pathé réalise même «un tour du monde léthal » (A. Montagne) : exécutions en Espagne, Amérique, Chine, etc.

30. La circulaire du 11 janvier 1909.

31. On pense ainsi au film de Victorin Jasset, La Bande de l'Auto Grise (1912), inspiré des méfaits de la bande à Bonnot.

32. Les « spectacles cinématographiques ne rentrent pas dans [la catégorie des] représentations d'ouvrages dramatiques dans le sens de la loi, mais bien plutôt dans [la] catégorie dits «de curiosités » visés par l'article 6 du décret du 6 janvier 1864 relatif à la liberté de l'industrie théâtrale » (circulaire du 11 janvier 1909). On notera qu'en 1864 , le cinéma n'existait pas encore.

33. Voir la brochure publicitaire déjà citée.

\section{RÉSUMÉS}

L'année 1913, avec l'adaptation cinématographique de Fantômas par Louis Feuillade, marque un moment charnière de la culture de masse en France, celui où le cinéma s'affirme comme un spectacle de masse à part entière. Fantômas - À l'ombre de la guillotine illustre en effet un double mouvement d'émancipation du cinéma à l'égard, d'une part, de la littérature populaire, et, d'autre part, du théâtre - moment d'émancipation complexe puisque, dans le même temps, Louis Feuillade inscrit son film dans la filiation de l'œuvre de Pierre Souvestre et Marcel Allain, et n'hésite pas à se tourner vers l'esthétique théâtrale pour traduire cinématographiquement des procédés littéraires. 
INDEX

Mots-clés : adaptation, affiche, censure, droit du cinéma, Fantômas, Feuillade Louis, Gaumont, générique, spectacle de curiosités

\section{AUTEUR}

\section{BÉRENGÈRE VACHONFRANCE-LEVET}

Bérengère Vachonfrance-Levet est titulaire d'une licence d'histoire et d'une maîtrise en lettres et civilisations étrangères (Anglais) de l'université Nice-Sophia-Antipolis. Tutrice à la TéléUniversité du Québec, elle a été assistante de recherche sur le projet CRSH » Fortunes médiatiques du roman populaire en France » dirigé par le Professeur P. Bleton (Téluq). Elle a publié « Roman Monde : Hédi Kaddour's Waltenberg », Paradoxa 24 (2012). 